\title{
CRECIMIENTO Y DESARROLLO INFANTIL TEMPRANO
}

\begin{abstract}
Melitón Arce ${ }^{1, a}$
RESUMEN

En este artículo se describen y comentan aspectos vinculados con el proceso de crecimiento y desarrollo del niño, poniendo énfasis en los primeros años de vida, periodo en el cual este proceso alcanza velocidad crítica en las estructuras y funciones más importantes de la economía humana. Se reafirma que ello puede contribuir a que la sociedad disponga de una generación cada vez mejor de adultos, los que a su turno serán capaces de contribuir a construir un mundo mejor y dentro de él una sociedad que goce de mayor bienestar. En un primer capítulo se mencionan consideraciones de orden general sobre la evolución favorable de la sociedad de humanos, sustentada en la calidad de sus futuros adultos, lo que equivale a decir de los logros que alcancen sus actuales niños. Un segundo capítulo menciona los aspectos básicos sobre crecimiento y desarrollo, en las diferentes esferas y los diversos fenómenos que en él se dan. En el tercero hacemos referencia a las oportunidades perdidas y los factores negativos que pueden incidir retrasando el proceso y con ello dando lugar a no obtenerse los logros esperados, en el cuarto se presentan las conclusiones y recomendaciones ratificando la concepción inicial de que un buen cuidado temprano del niño sirve para construir una sociedad mejor, y se formulan algunas recomendaciones para hacer del mismo una buena práctica.
\end{abstract}

Palabras clave: Desarrollo infantil; Niño; Crecimiento y desarrollo (fuente: DeCS BIREME).

\section{EARLY CHILDHOOD GROWTH AND DEVELOPMENT}

\begin{abstract}
This article describes and discusses issues related to the process of childhood growth and development, with emphasis on the early years, a period in which this process reaches critical speed on major structures and functions of the human economy. We reaffirm that this can contribute to the social availability of a generation of increasingly better adults, which in turn will be able to contribute to building a better world and within it a society that enjoys greater prosperity. In the first chapter, we discuss the general considerations on the favorable evolution of human society based on quality of future adults, meaning the accomplishments that today's children will gain. A second chapter mentions the basics of growth and development in the different fields and the various phenomena that occur in it. In the third we refer to lost opportunities and negative factors that can affect delaying the process and thereby result in not obtaining the expected accomplishments. In the fourth, conclusions and recommendations are presented confirming the initial conception that good early child care serves to build a better society and some recommendations are formulated to make it a good practice.
\end{abstract}

Key words: Child development; Child; Growth and development (source: MeSH NLM).

\section{INTRODUCCIÓN}

Desde su aparición sobre la tierra, el hombre fue sin duda el indiscutible autor y actor de su desarrollo $y$, por ende, de su propio destino. Abonan en ese sentido los acontecimientos históricos que se han podido registrar y que de diversa manera, y con concepciones variadas, han sido recogidas en las tres más importantes teorías aceptadas sobre la evolución de las especies: (a) la primera que se formuló, conocida como de la adaptación o del transformismo, que postula el progresivo perfeccionamiento de las estructuras y funciones biológicas, orientadas a satisfacer las nuevas y crecientes exigencias planteadas por la necesidad de supervivencia, que fuera patrocinada por Jean Baptiste Lamarck; (b) la de la selección natural, que postula la evolución en base a la sobrevivencia de los más fuertes y con ello la mejora continua -optimización- en las capacidades humanas $\mathrm{y}$, consecuentemente, de una mayor disponibilidad de recursos para sobrevivir de las sucesivas generaciones, preconizada por Charles Darwin, y (c) la de la mutación genética, que

\footnotetext{
Academia Nacional de Medicina. Lima, Perú

a Médico cirujano, doctor en medicina, exjefe del Departamento de Medicina del Instituto Nacional de Salud del Niño, profesor emérito de la Universidad Federico Villareal, académico honorario de la Academia Nacional de Medicina

Recibido: : 24-07-15 Aprobado: 16-09-15
}

Citar como: Arce M. Crecimiento y desarrollo infantil temprano. Rev Peru Med Exp Salud Publica. 2015;32(3):574-8. 
admite la aparición periódica, imperceptible, de nuevas características, como consecuencia de modificaciones en el patrón genético humano, sustentada por Hugo Marie De Vries ${ }^{(1-3)}$

Resultado de las consideraciones precedentes es una primera conclusión, en el sentido que fue el espíritu de supervivencia el que llevó al hombre, a través de los siglos, a generar cada vez mayores instrumentos y capacidades para aprovechar en mayor medida las oportunidades y los recursos que le garantizaran una vida mejor, y una segunda, en el hecho de que fue con el mismo propósito que se preocupó por vivir en comunidad, buscando en ella las seguridades que bajo el principio de reciprocidad demandaban una mutua protección, al aprovechar mejor las diferentes capacidades de cada uno de sus miembros.

Este primer análisis confirma, pues, la validez de nuestra afirmación inicial, que lo logrado hasta hoy por la humanidad es obra del hombre que supo, a través del tiempo, imponerse a su circunstancia. De este modo nacieron y fueron creciendo las sociedades, que pasaron progresivamente de clanes a tribus y a conglomerados mayores, permitiendo la construcción de lo que son hoy, en su expresión mayor las naciones y los estados.

Para que pudiera suceder lo anteriormente expuesto tenemos que admitir que, a su turno, cada generación de humanos tuvo que contribuir a tal fin mejorando la calidad de su descendencia -pues no de otro modo podría haberse logrado construir este futuro mejor de la sociedad - al permitir a ella un despliegue cada vez mayor de su potencial genético, aplicando, dentro de las limitadas posibilidades que el insuficiente avance que la ciencia de su época podía ofrecerle, los mejores cuidados posibles. Las sucesivas generaciones fueron así conociendo mayores posibilidades de mejora y alcanzando mayores posibilidades de cuidar de sus niños y, con ello, de disponer de poblaciones adultas con creciente capacidad para satisfacer sus necesidades para que de ese modo se logre mayor bienestar ${ }^{(4,5)}$.

Fueron surgiendo entonces, lenta, pero inexorablemente, comunidades más evolucionadas y prósperas y, en ellas, los niños fueron recibiendo cada vez mejores cuidados -la lactancia materna tuvo que haber sido fundamental- y pudieron ser mejor instruidos para que, cuando adultos, superaran a sus padres y así elevaran el nivel de vida de los suyos. Conforme estas limitaciones iban siendo superadas se fue valorando, cada vez más, la trascendencia del potencial genético que cada niño traía al nacer y la importancia de permitir a este su plena expresión y conociendo también cada vez mejor la importancia del indispensable concurso de los factores ambientales que lo facilitaran.
Fue entonces quedando claro la importancia que una niñez saludable tenía para garantizar un mejor devenir a las respectivas sociedades, convicción aceptada hoy en el aforismo universalmente admitido "que los niños constituyen el futuro de la humanidad", porque cuando adultos podrán labrar mejores destinos y que de las competencias que adquieran en la etapa temprana de su vida dependerá el advenimiento de una sociedad mejor.

Resultó así de necesidad fundamental atender debidamente la salud del niño, para permitirle acceder a la adultez premunido de mayores competencias, necesarias y suficientes para contribuir al mejoramiento de su generación y para sentar las bases de mejores generaciones futuras que continuarán construyendo un mundo que cada vez brinde a la humanidad una mejor calidad de vida.

\section{ASPECTOS BÁSICOS SOBRE CRECIMIENTO Y DESARROLLO DEL NIÑO}

El niño crece y desarrolla desde la concepción, es la unión del espermatozoide y el óvulo la que marca su inicio. Es un proceso, en condiciones normales, continuo, pero de velocidad variable según las diversas edades y las diferentes estructuras orgánicas, alcanzando su máximo crecimiento en diferentes momentos; así, por ejemplo, mientras que el cerebro crece un $90 \%$ en los primeros 5 años y los pulmones requieren 8 años para alcanzar su estructura definitiva, la altura final se logra recién al concluir la adolescencia ${ }^{(6)}$.

Por otra parte, es importante recordar que son tres los fenómenos que caracterizan el proceso de crecimiento y desarrollo del niño; (a) cambios en magnitud, es decir, aumento de tamaño, tanto del cuerpo en su totalidad como de los diferentes órganos en particular; (b) cambios en características, como por ejemplo la extinción de los reflejos del recién nacido y su sustitución por movimientos intencionales y los que se observan en aspecto somático, y (c) perfeccionamiento de las estructuras y funciones, fenómeno, este último que se conoce como maduración. Durante todos ellos se produce incremento del número de células, modificaciones en estas de acuerdo con la función específica que habrán de realizar y su migración dentro del órgano o aparato al que pertenecen, para consolidar la arquitectura de los mismos Estos fenómenos ocurren simultáneamente, aunque con ritmos también diferentes para cada órgano, aparato y sistema ${ }^{(7,8)}$.

Respecto a los cambios en magnitud, si bien cobra la mayor atención el que se refiere al aumento de tamaño del niño, por ser el más fácil de observar y de evaluar, 
no debe ignorarse que, simultáneamente, también, los demás órganos de la economía humana están creciendo y que cada uno de ellos tiene su propia cronología y velocidad, haciéndolo, la mayoría, en los primeros años de vida, tampoco debe ignorarse que la evolución normal de ellos es fundamental para garantizar el crecimiento integral del niño. En la esfera motora observamos este aumento en magnitud cuando comprobamos que el niño, que al principio tiene solamente movimientos reflejos y unos pocos intencionales, va incrementando el número de estos últimos hasta tener la capacidad de caminar y desplazarse libre y voluntariamente, y en lo psicoemocional, cómo el niño incrementa su capacidad de interactuar con el medio y las personas que lo rodean, pasando del llanto a la sonrisa, del balbuceo a los monosílabos y finalmente a la palabra ${ }^{(9)}$.

Respecto a los cambios en características, se aprecia cómo progresivamente la proporción entre las dimensiones de las diferentes partes del cuerpo van variando e, incluso, los órganos internos van ubicándose en forma más precisa dentro de la localización que, anatómicamente, le corresponde; por poner solo un ejemplo, recordemos que el hígado y el bazo, al principio fácilmente palpables, dejan de serlo conforme el crecimiento de la parrilla costal los cubre; en el sistema óseo se observa el cierre progresivo de las fontanelas del cráneo, la aparición de los núcleos y los cartílagos de crecimiento y su posterior osificación, asimismo, el mayor desarrollo muscular y con ello la fuerza consiguiente, no menos importantes los cambios psicoemocionales que transitan desde la imaginación fantástica a la realística, del egocentrismo al deseo de compartir con otros niños y progresivamente al desarrollo de un mayor espíritu gregario.

El tercer fenómeno es el del perfeccionamiento de las estructuras y funciones, ocurre, como queda dicho, simultáneamente con los dos anteriores y se traduce en la capacidad creciente que va desarrollando el niño tanto en las que le permiten una vida de relación cada vez más rica y fructífera, como en las que determinan un mayor control de su mundo interno y autonómico, como son los ritmos circadianos y los equilibrios homeostático, metabólico e inmunológico, entre otros.

Desde un punto de vista funcional los fenómenos descritos pueden pues agruparse en tres esferas, la primera es la esfera física, referida a los cambios en las dimensiones y características tanto somáticas como viscerales, ya descritas; la segunda, la esfera motora que tiene que ver con la capacidad creciente de movimientos y su coordinación y que comprende a la vez tanto la función motora gruesa, que sirve al desplazamiento corporal, como la motora fina, que se relaciona con el desarrollo de habilidades y, la tercera, la psicoemocional que engloba el lenguaje, la inteligencia y las emociones. Ahora bien, los cambios que se producen en cada una de estas esferas no ocurren independientemente unas de otras, sino que se dan simultánea y coordinadamente, guardando entre sí estrecha correspondencia e interacción. A pesar de ello, es posible evaluar su progresión, a través de indicadores, los que permiten detectar retrasos y deficiencias y una vez determinada su causa corregirlas para que el proceso continúe sin mayor afectación (10-12). El cuadro siguiente pretende esquematizar lo expuesto

\section{CAMBIOS QUE CARACTERIZAN EL PROCESO DE CRECIMIENTO Y DESARROLLO DEL NINO}

\section{A NIVEL CELULAR}

- Incremento de su número.

- Modificaciones de su estructura de acuerdo a la función prevista.

- Migración en el interior de cada órgano, aparato y sistema a efecto de consolidar la estructura definitiva.

\section{EN CADA ÓRGANO, APARATO O SISTEMA}

- Cambios en magnitud: incremento de sus dimensiones, salvo en algunas estructuras que con el tiempo la reducen o desaparecen.

- Cambios en características: variaciones en todos ellos conforme van alcanzando su dimensión y estructura final.

- Perfeccionamiento de las funciones: cada uno va alcanzando la competencia funcional que le corresponde; proceso también denominado "maduración".

\section{ESFERAS EN LAS QUE SE APRECIAN LOS CAMBIOS PRODUCIDOS}

Esfera física: cambios en los diferentes órganos, en cuanto a la dimensión de sus diferentes segmentos y las proporciones que guardan entre sí, así como a un mayor desarrollo de su capacidad para el desempeño de sus diferentes funciones, el que se observa tanto en el ámbito somático como visceral.

Esfera motora: desaparición de la actividad motora refleja inicial, propia del neonato, y aparición progresiva de la motilidad intencional asociada con un desarrollo creciente del aparato locomotor que permite una mayor capacidad de desplazamiento y el desarrollo de habilidades manuales. 
Esfera psicoemocional: la forma única e inicial de expresar sus emociones, el llanto, se va enriqueciendo con la capacidad de sonreír, balbucear y hablar y, simultáneamente, de demostrar preferencia hacia las personas de su entorno cercano, la cual, progresivamente se dirigirá a una socialización mayor; a ello se agrega el tránsito desde la imaginación fantástica a la realística, y del egocentrismo a la capacidad de compartir.

Conviene también recordar otras características del niño, sin duda también vinculadas al proceso de crecimiento y desarrollo que hemos señalado como la característica principal; ellas son la vulnerabilidad y la dependencia, ambas en grado inverso a su edad y que obedecen a la inmadurez relativa de sus capacidades, tanto para controlar su mundo interno como el de relación y que obligan a darles mayor protección. Por la primera de ellas habrá de requerir ser protegido de las noxas ambientales de todo orden, las infecciones entre ellas, por la segunda, los peligros externos -las llamadas externalidades- al no disponer de suficiente capacidad de discernimiento y de discriminación para evitar peligros ni las competencias para alejarse de ellas.

\section{OPORTUNIDADES PERDIDAS}

El crecimiento y desarrollo del niño se sustenta en dos pilares fundamentales: el patrón genético, esto es el total de genes que a través de los padres le han sido transferidos y los factores ambientales que inciden en que aquellos se expresen libre y plenamente, o no lo hagan; entre estos últimos distinguimos dos grupos, de un lado, los que conocemos como factores ambientales externos, entre ellos la alimentación apropiada para satisfacer sus necesidades en cada etapa de su vida; la estimulación permanente a través de sus sentidos, que están en progresiva evolución, y también del aporte afectivo, social y emocional que le prodiguen quienes lo rodean y, desde luego, garantizarles la ausencia de enfermedades, eventos que por afectar su nutrición, por exacerbar su catabolismo o aislarlo temporalmente de su medio, le privan de la oportunidad de avanzar en este proceso; por otro lado están los factores ambientales internos, es decir, el equilibrio del medio interno, tan importante para las funciones de asimilación nutricional, para la óptima respuesta inmunológica así como su capacidad metabólica. Atender estos aspectos y fomentar su benéfica acción es, en consecuencia, indispensable. Esto ha calado actualmente en la sociedad y en su favor se desarrollan importantes programas de información, promoción, apoyo y asistencia ${ }^{(13,14)}$.

De no atenderse aquello se expone al niño a la pérdida de oportunidades. Recordemos que la expresión de los genes $\mathrm{y}$, consecuentemente, la aparición y desarrollo de los caracteres y funciones que comandan, se dan en momentos diversos, y por ello el concurso de tales factores debe ser permanente y estar presente cuando lo genético lo requiera; de no ocurrir así, el crecimiento y desarrollo del niño resultará afectado en esa esfera; esto es lo que conocemos como oportunidad perdida y lo que resulta grave es que nunca puede medirse con criterio prospectivo y, en consecuencia, conocerse el grado exacto de afectación. Algunos ejemplos nos permitirán clarificar lo que afirmamos: si un niño enferma y requiere hospitalización, digamos, por 1 mes, durante ese tiempo permanecerá aislado y su medio interno alterado; consecuentemente, corre el riesgo, en ese lapso, de perder en nutrición, sufrir alteraciones metabólicas persistentes y dejar de ser estimulado, es decir, de perder un mes de oportunidades. Luego se recuperará, pero no podemos definir en qué medida ni cuanto perdió por esa causa, a pesar de que continúe aparentando normalidad. Los casos más dramáticos se viven en la esfera del crecimiento físico tratándose de la altura -se podrá decir que a pesar de haber estado enfermo durante largo tiempo logró al final una talla de $1,65 \mathrm{~m}$, pero nadie podrá saber si acaso debería haber alcanzado 1,70 o algo más; lo mismo ocurre en la esfera psicoemocional, en la que la mayoría de nuestro niños, en el pasado, han alcanzado coeficientes intelectuales promedio, pero ¿cuántos de ellos pudieron haber desarrollado promedios mayores de no haber enfermado tan grave y/o frecuentemente y no haber sufrido hospitalizaciones prolongadas?.

Cuando me desempeñaba como profesor de pediatría en el pregrado, solía graficar este aspecto a los alumnos, comparando el crecimiento y desarrollo del niño con el ascenso por una escalera, teniendo en consideración que cada peldaño era una oportunidad para aprovechar mejor el potencial genético. De no encontrar en alguno de ellos los factores ambientales necesarios para lograrlo, les decía, el niño pierde esa oportunidad y mientras los demás avanzan a su ritmo normal, él va quedando relegado, sumiéndonos en la duda de si alcanzará la cima o si llegará a ella más tarde y con limitaciones.

De lo anterior resulta trascendente que la sociedad en su conjunto, las instituciones vinculadas al quehacer de la salud, los profesionales del área, los maestros y, sobre todo, los padres, se preocupen por brindar al niño todas las garantías necesarias para alcanzar un óptimo grado de crecimiento y desarrollo,

\section{CONCLUSIONES Y RECOMENDACIONES}

De lo anteriormente expuesto queda claro que cuidar del niño y garantizarle un óptimo crecimiento y desarrollo durante toda la etapa que constituye la niñez, en 
particular en los primeros años de vida, es fundamental si se aspira a convertirlos en adultos con capacidad suficiente para mejorar el nivel de vida y el bienestar de la sociedad a la que han accedido, y que ello pasa por permitir que el potencial genético de cada cual tenga la oportunidad de expresarse plenamente en base al concurso de los factores condicionantes externos e internos referidos.

Para lograr ello recomendamos como indispensable aplicar las siguientes medidas:

Promover gestaciones programadas y saludables, con suficiente y eficaz control prenatal, y la atención segura de parto, de preferencia institucional, en el momento actual.

Proveer alimentación apropiada a cada edad, destacando en este campo, la lactancia materna exclusiva desde el instante mismo del nacimiento y durante el primer semestre, pudiendo continuarla asociada con una buena alimentación no láctea durante los 2 primeros años de vida.
Llevar a cabo programas eficientes de prevención y promoción de la salud a través de intervenciones costoefectivas y de educación sanitaria de carácter integral.

Garantizar el diagnóstico y la atención oportuna y eficiente de las enfermedades, reduciendo al mínimo indispensable la estancia hospitalaria.

Equipar suficientemente los establecimientos de salud, dotándolos del personal necesario.

Llevar la escuela al hospital, esto es, mantener la actividad escolar en los ambientes de hospitalización, para que los niños en capacidad de recibir clases, lo hagan.

Educar a los padres para un óptimo cuidado de la salud de sus hijos, poniendo énfasis en los futuros padres.

Fuentes de financiamiento: autofinanciado.

Conflictos de interés: el autor refiere no tener conflictos de interés.

\section{REFERENCIAS BIBLIOGRÁFICAS}

1. Lamarck JB. Philosophie zoologique, ou exposition des considérations relatives à l'histoire naturelle des animaux... Paris: Duminil-Lesueur; 1809.

2. Darwin C. On the Origin of the Species. London; 1859.

3. de Vries $\mathrm{H}$. The mutation theory. Chicago: Open Court Publishing Company; 1909.

4. Taylor AR. El Estudio del Niño: breve tratado de la psicología del niño con indicaciones a los maestros, estudiantes y padres de familia. Nueva York: D. Appleton, 1913.

5. Holt Jr, MR, Barnett HL. Growth and development in pediatrics. 13th ed. New York: D. Apletton Century Co Inc.; 1961.
6. Turkin Salmy Ts. Crecimiento y desarrollo del niño normal. En: Fanconi G, Wallgren A. Pediatría. Madrid: Morata; 1961.

7. Rousseau JJ. Emilio. Lima: La Confianza; 1971.

8. González S. Crecimiento y Desarrollo. En: Meneghello J. Pediatría. Buenos Aires: Intermédica; 1972.

9. Polonovski C. Crecimiento Somático. En: R. Laplanne. Manual de Pediatría. Barcelona: Toray Mason; 1972.

10. Davis JA, Dobbing J. Scientific Foundations of Paediatrics, 2nd ed. London, UK: William Heinemann Medical Books; 1981.

11. Cruz Hernández M. Tratado de Pediatría, $7 \mathrm{ma}$ ed. Barcelona: ESPAXI Pub.Médicas; 1983.
12. Arce Rodríguez M. Constantes fisiológicas y clínicas en el niño. Actualización Clínica Continua. 2da Ed. Lima: Abeefe; 1997.

13. Perú, Ministerio de Salud. Esquema de Desarrollo del Niño. CONAII-INR: MINSA; 1993.

14. Perú, Ministerio de Salud. Norma Técnica de Salud para el Control de Crecimiento y Desarrollo de la Niña y el Niño menor de 5 años. Lima: MINSA; 2011.

Correspondencia: Melitón Arce Teléfono:(+511) 999419096

Correoelectrónico:serctec@terra.com.pe 